\title{
Neck muscle vibration induces lasting recovery in spatial neglect
}

\author{
I Schindler, G Kerkhoff, H-O Karnath, I Keller, G Goldenberg
}

See Editorial Commentary, page 357

J Neurol Neurosurg Psychiatry 2002;73:412-419

See end of article for authors' affiliations

...................

Correspondence to: Dr I Schindler, Cognitive Neuroscience Research Unit, Wolfson Research Institute, University of Durham Stockton Campus, University Boulevard,

Stockton-on-Tees

TS17 6BH, UK;

igor.schindler@durham.ac.uk

Received 14 August 2001 In revised form 19 April 2002

Accepted 23 May 2002
Objectives: To evaluate whether neck muscle vibration is an effective technique for neglect rehabilitation, with lasting beneficial effects.

Methods: The effects of differential treatment of visual exploration training alone or in combination with neck muscle vibration were evaluated in a crossover study of two matched groups of 10 patients suffering from left sided neglect. Each group received a sequence of 15 consecutive sessions of exploration training and combined treatment. The effects of treatment were assessed with respect to different aspects of the neglect disorder such as impaired perception of the egocentric midline, exploration deficits in visual and tactile modes, and visual size distortion. The transfer of treatment effects to activities of daily living was examined by a reading test and a questionnaire of neglect related everyday problems. All variables were measured six times: three baseline measurements, two post-treatment measurements, and one follow up after two months.

Results: The results showed superior effects of combination treatment. A specific and lasting reduction in the symptoms of neglect was achieved in the visual mode, which transferred to the tactile mode with a concomitant improvement in activities of daily living. The improvement was evident two months after the completion of treatment. In contrast, isolated exploration training resulted in only minor therapeutic benefits in visual exploration without any significant transfer effects to other tasks.

Conclusions: Neck muscle vibration is a decisive factor in the rehabilitation of spatial neglect and induces lasting recovery when given as a supplement to conventional exploration training.
S patial neglect is a multimodal neurological disorder in which patients fail to acknowledge, orient, or react appropriately to stimuli located on the contralesional side of space. ${ }^{12}$ Spatial neglect can be observed in $50 \%$ of patients who suffer predominantly right hemisphere brain damage. ${ }^{3}$ As the main symptom of neglect is a defective exploration of contralesional space, the standard clinical treatment of neglect aims to enhance visual exploration by training the patient to make saccadic eye movements to the impaired field, thereby improving visual scanning and utilisation of compensatory search strategies..$^{4-6}$ Although this treatment is successful in the management of patients with postchiasmatic visual field disorders, ${ }^{7}$ its efficacy in neglect remains controversial. While some studies report positive effects, ${ }^{48}$ others showed only limited improvement, even after intensive therapy, and this rarely generalised beyond the specific tasks being trained. ${ }^{6}{ }^{10}$

A major limitation of this method is that it requires patients to have some awareness of their deficit in order to compensate actively for their right sided orientational bias. Therefore more recent studies have focused on alternative techniques derived from current theoretical accounts of spatial neglect. These include sustained attention training ${ }^{11}{ }^{12}$ and various techniques of basic sensory stimulation. In relation to the latter, substantial reductions in neglect symptoms have been achieved by vestibular stimulation, ${ }^{1314}$ optokinetic stimulation, ${ }^{15} 16$ neck muscle vibration, ${ }^{17}{ }^{18}$ prism adaptation, ${ }^{19}{ }^{20}$ contralesional limb activation, ${ }^{12}{ }^{21-24}$ and trunk rotation. ${ }^{17}{ }^{25}$ Although successful in their immediate applications, the long term potential of these stimulation methods remains unclear in many cases. Therapeutic effects leading to a generalised improvement in the activities of daily living lasting for at least one month have only been demonstrated for trunk rotation, ${ }^{25}$ limb activation training, ${ }^{21} 23$ and prism adaptation. ${ }^{19}$

A further promising candidate for potential long term rehabilitation effects is neck muscle vibration. In the normal system, it has been shown that the deviation of subjective straight ahead perception induced by prolonged asymmetrical neck vibration is not subject to habituation and has effects that endure after the vibration terminates. ${ }^{26}$ Neck muscle vibration is non-invasive, has no side effects, and is technically easy to apply. A pilot study carried out with a single patient with neglect provided promising results in a visual exploration task. ${ }^{27}$

The approach to the use of basic sensory stimulation for manipulating spatial neglect is based on anatomical studies in human ${ }^{28}$ and neurophysiological work on the primate brain. These studies suggest that the posterior parietal lobe is a multimodal association area, forming a distributed representation of space. ${ }^{29-31}$ One model attributes the multimodal character of neglect to damage to a sensory integration area where the inputs of peripheral sensory and motor related signals become integrated into an egocentric coordinate system responsible for the localisation of the body in space, and of object position relative to the body-both necessary for appropriate spatial behaviour. ${ }^{32} 33$ According to this model the peripheral sensory representation systems are deemed largely intact, whereas the transformation to the highest level of supramodal representation is subject to a systematic ipsilesional error, thus leading to an extreme rightward orientation bias of patients with left sided neglect. ${ }^{173}$

To evaluate whether neck muscle vibration is an effective technique for neglect rehabilitation-that is, whether it is capable of producing lasting beneficial effects-we compared the long term efficacy of combined vibration/exploration treatment with visual exploration training alone.

Abbreviations: $A D L$, activities of daily living; CS/SC, order of treatment: combination (C) followed by standard (S) or vice versa; PC, personal computer; SSA, subjective visual straight ahead test; SSA $_{v}$, SSA deviation during vibration 
Table 1 Demographic and clinical data of the 20 neglect patients according to treatment sequence (CS, combination-standard; SC, standard-combination)

\begin{tabular}{|c|c|c|c|c|c|c|c|c|c|c|c|c|c|c|c|c|}
\hline \multirow[b]{2}{*}{ Patient } & \multirow{2}{*}{$\begin{array}{l}\text { Age } \\
\text { (years) }\end{array}$} & \multirow{2}{*}{ Sex } & \multirow{2}{*}{$\begin{array}{l}\text { Education } \\
\text { (years) }\end{array}$} & \multirow{2}{*}{$\begin{array}{l}\text { Time of } \\
\text { onset } \\
\text { (months) }\end{array}$} & \multicolumn{2}{|l|}{ Lesion } & \multirow{2}{*}{$\begin{array}{l}\text { Visual field } \\
\text { defect, sparing } \\
\left({ }^{\circ}\right)\end{array}$} & \multirow[b]{2}{*}{ Motricity } & \multicolumn{4}{|c|}{ Line bisection* } & \multicolumn{2}{|c|}{ Copying } & \multicolumn{2}{|c|}{ Drawing } \\
\hline & & & & & Aetiology & Site & & & $\mathrm{L}$ & $M$ & $R$ & MPD & L & $\mathbf{R}$ & L & $\mathbf{R}$ \\
\hline \multicolumn{17}{|c|}{ Group CS } \\
\hline WK & 43 & $M$ & 17 & 4 & I & $F, T, P, S C$ & $\mathrm{HA}, 4^{\circ}$ & Paresis & 3 & 0 & 0 & 43.42 & $2 / 5$ & $0 / 9$ & 3 & 2 \\
\hline $\mathrm{FE}$ & 30 & $\mathrm{~F}$ & 10 & 5 & $\mathrm{H}$ & T,P,SC & $\mathrm{HH}, 2^{\circ}$ & Plegia & 2 & 0 & 0 & 39.86 & $5 / 5$ & $2 / 9$ & 3 & 1 \\
\hline SK & 51 & $M$ & 10 & 4 & 1 & $\mathrm{~T}, \mathrm{P}, \mathrm{SC}$ & $Q A, 50^{\circ}$ & Paresis & 1 & 0 & 0 & 29.67 & $4 / 5$ & $0 / 9$ & 3 & 1 \\
\hline $\mathrm{HB}$ & 52 & $\mathrm{~F}$ & 10 & 13 & $\mathrm{H}$ & $F, T, P, O, S C$ & $\mathrm{HH}, 5^{\circ}$ & Paresis & 5 & 2 & 0 & 50.92 & $5 / 5$ & $1 / 9$ & 3 & 1 \\
\hline$B C$ & 49 & $\mathrm{~F}$ & 11 & 3 & 1 & $\mathrm{~T}, \mathrm{SC}$ & $\mathrm{HH}, 3^{\circ}$ & Paresis & 5 & 4 & 0 & 70.70 & $5 / 5$ & $3 / 9$ & 3 & 2 \\
\hline GK & 59 & $M$ & 17 & 3 & i & $\mathrm{T}, \mathrm{SC}$ & $\mathrm{HH}, 3^{\circ}$ & Paresis & 4 & 1 & 0 & 71.95 & $5 / 5$ & $3 / 9$ & 3 & 2 \\
\hline SU & 48 & $\mathrm{~F}$ & 10 & 3.5 & $\mathrm{H}$ & $\mathrm{F}, \mathrm{T}, \mathrm{SC}$ & $\mathrm{HH}, 2^{\circ}$ & Paresis & 4 & 2 & 0 & 59.81 & $4 / 5$ & $0 / 9$ & 3 & 2 \\
\hline WA & 78 & $M$ & 8 & 2 & $\mathrm{H}$ & T,WM & Normal, $63^{\circ}$ & Plegia & 2 & 1 & 0 & 46.32 & $1 / 5$ & $0 / 9$ & 1 & 0 \\
\hline $\mathrm{KJ}$ & 52 & $M$ & 10 & 2 & I & $F, T, P, O, S C$ & $\mathrm{HH}, 3^{\circ}$ & Paresis & 6 & 3 & 1 & 63.00 & $5 / 5$ & $1 / 9$ & 3 & 2 \\
\hline $\mathrm{OE}$ & 24 & M & 9 & 5.5 & $\mathrm{Tr}$ & $\mathrm{T}, \mathrm{P}, \mathrm{WM}$ & $\mathrm{HH}, 4^{\circ}$ & Paresis & 2 & 0 & 0 & 51.38 & $3 / 5$ & $2 / 9$ & 2 & 1 \\
\hline \multicolumn{17}{|c|}{ Group SC } \\
\hline RM & 56 & $M$ & 8 & 2 & 1 & $\mathrm{~T}, \mathrm{P}, \mathrm{WM}$ & Normal, $74^{\circ}$ & Paresis & 5 & 3 & 1 & 43.51 & $3 / 5$ & $0 / 9$ & 3 & 2 \\
\hline DR & 20 & $M$ & 12 & 30 & $\mathrm{Tr}$ & $F, T, P, O, S C$ & $\mathrm{HH}, 2^{\circ}$ & Paresis & 1 & 0 & 0 & 17.28 & $2 / 5$ & $0 / 9$ & 2 & 2 \\
\hline PK & 51 & $M$ & 9 & 5 & 1 & $\mathrm{~T}, \mathrm{IC}, \mathrm{S}$ & $\mathrm{HH}, 3^{\circ}$ & Paresis & 2 & 1 & 0 & 23.00 & $1 / 5$ & $0 / 9$ & 2 & 1 \\
\hline MA & 57 & $\mathrm{~F}$ & 12 & 3 & 1 & $\mathrm{O}, \mathrm{IC}, \mathrm{S}, \mathrm{WM}$ & $\mathrm{HH}, 2^{\circ}$ & Paresis & 4 & 4 & 0 & 63.96 & $3 / 5$ & $1 / 9$ & 3 & 1 \\
\hline KC & 60 & $\mathrm{~F}$ & 10 & 2 & $\mathrm{H}$ & $T, P, I C$ & $\mathrm{QA}, 10^{\circ}$ & Paresis & 3 & 0 & 0 & 51.07 & $3 / 5$ & $0 / 9$ & 2 & 0 \\
\hline MK & 30 & $\mathrm{~F}$ & 13 & 5 & $\mathrm{Tr}$ & $F, T, P, O, S C$ & $\mathrm{HH}, 5^{\circ}$ & Paresis & 6 & 2 & 1 & 63.70 & $5 / 5$ & $2 / 9$ & 3 & 2 \\
\hline SJ & 58 & $\mathrm{~F}$ & 16 & 2.5 & 1 & $T, P, S C$ & $\mathrm{HH}, 3^{\circ}$ & Paresis & 4 & 1 & 0 & 47.31 & $2 / 5$ & $1 / 9$ & 2 & 1 \\
\hline $\mathrm{TH}$ & 65 & $\mathrm{~F}$ & 10 & 2 & 1 & $\mathrm{~T}, \mathrm{P}, \mathrm{O}, \mathrm{SC}$ & $\mathrm{HH}, 5^{\circ}$ & Plegia & 5 & 2 & 0 & 68.32 & $5 / 5$ & $2 / 9$ & 2 & 1 \\
\hline MM & 38 & $\mathrm{~F}$ & 10 & 2.5 & $\mathrm{H}$ & $F, T, P, S C$ & $\mathrm{HH}, 12^{\circ}$ & Paresis & 0 & 0 & 0 & 43.33 & $5 / 5$ & $2 / 9$ & 3 & 1 \\
\hline KM & 53 & $\mathrm{~F}$ & 9 & 2 & $\mathrm{H}$ & $\mathrm{T}, \mathrm{P}, \mathrm{SC}$ & $Q A, 10^{\circ}$ & Paresis & 4 & 1 & 0 & 58.99 & $5 / 5$ & $2 / 9$ & 3 & 2 \\
\hline
\end{tabular}

*Schenkenberg: omissions (L, M, R) and MPD.

†Rey-Osterrieth figure.

$\ddagger$ Scores according to Schenkenberg from $0=$ unimpaired to $3=$ severely impaired.

$\mathrm{F}$, frontal; $\mathrm{H}$, haemorrhage; $\mathrm{HA}$, hemiamblyopia; $H \mathrm{H}$, homonymous hemianopia; I, ischaemia; IC, internal capsule; $L$, left; $M$, middle; $M P D$, mean

percentage deviation; O, occipital; P, parietal; QA, quadranopia; R, right; S, striatum; SC, subcortical; T, temporal; Tr, head trauma; WM, white matter.

\section{METHODS}

\section{Subjects}

Twenty patients with right sided brain damage and left hemispatial neglect participated in the study (table 1). They had been admitted to a neurological rehabilitation hospital for persistent neglect and moderate to severe hemiparesis. All were right handed according to the Edinburgh inventory. ${ }^{34} \mathrm{~A}$ unilateral hemispheric lesion was documented in every case by computed tomography or magnetic resonance imaging.

None of the patients suffered from impaired vigilance for up to one hour, confusion, general mental deterioration, or psychiatric disorders, and none had a history of previous neurological disease. All patients gave their informed consent to participate in the study, which was approved by the local ethics committees of the neurological hospitals MunichBogenhausen and Bad Aibling.

Before the treatment all patients were screened using three neglect tests: line bisection, copying the Rey-Osterrieth figure, and drawing.

Line bisection and drawing followed the procedure of Schenkenberg et al. ${ }^{35}$ For line bisection, the overall number of omissions of the 18 lines and the average deviation score from the objective midline were determined. In the drawing task each patient was asked to draw a daisy, a clock face, a house, and a human face. Two neuropsychologists who were not involved in the study rated the quality of the drawings. The overall reliability between the ratings was $r=0.92$.

For the Rey-Osterrieth figure the Rapport scoring method was applied where only the numbers of omissions relating to items on the left and right side of the figure were noted. ${ }^{36}$ Items attributed to both sides were excluded from the analysis.

The first 10 of 20 consecutively admitted patients were assigned randomly to two groups with different treatment sequences. Because of the small sample size the remaining patients were allocated to these groups by matching the other members of the group as closely as possible for clinical and demographical variables, as shown in table 1 , to avoid any randomisation bias. The lesions sites were matched with respect to affected brain structures, following the Damasio templates scheme. ${ }^{37}$

There were no statistical differences in the clinical and demographical data of the two groups (Mann-Whitney U test $\geqslant 33.0, \mathrm{p}>0.05$ ).

\section{Neglect treatments}

\section{Standard treatment}

As a standard treatment condition we used visual exploration training. ${ }^{5}$ This consisted of five 40 minute sessions a week, composed of four different procedures carried out in a fixed sequence interspersed with short (five minute) breaks. The session started with the training of smooth pursuit eye movements towards the ipsilesional and contralesional side on the horizontal and diagonal visual axes. Patients were required to follow slow movements of a pencil performed by the therapist sitting in front of them.

This task was followed by two PC based saccadic eye movement training programmes to encourage fast and precise eye movements to the contralesional side without any head movement. In the first task a small randomly positioned red or green square $\left(0.5 \mathrm{~cm}^{2}\right)$ was presented on a PC screen. The patient was required to press one of two buttons to indicate, as rapidly as possible, which colour had been presented. In the second task, tachistoscopically presented single words of variable length (three to 11 letters) were presented on the horizontal axis and had to be read aloud. In both tasks the target eccentricity, duration, and predictability were adapted to the patient's individual ability and progress with respect to a training protocol. Task difficulty was increased when the patient achieved 75\% correct responses. Additionally, compensatory visual training on wide field visual displays was conducted to enlarge the size of the visual search field and improve visual search strategies in contralateral as well as ipsilateral hemispace. The patients were trained to adopt a systematic spatially organised search strategy (row by row or column by column), beginning on the neglected side. This training was based on 100 specifically designed slides with 
displays (eccentricity: $90^{\circ}$ horizontal $/ 60^{\circ}$ vertical), which varied in the overall number and size of stimuli and distractors, target to foil ratio, similarity among stimuli, and array configuration (systematic or mixed).

\section{Combined treatment}

In the combined treatment condition, the patients performed the same visual exploration training while the contralesional posterior neck muscles were vibrated. For stimulation, a vibrator (Gearing and Watson Electronics, Hailsham, East Sussex, UK) was used, with a frequency of $80 \mathrm{~Hz}$ and an amplitude of $0.4 \mathrm{~mm}$. The tip of the vibrator, a flat $2 \mathrm{~cm}$ diameter disk, was placed on the subject's contralesional posterior neck muscles. The exact position of the vibrator was individually adjusted to optimise the vibration induced shift of the perceived position of the body midline. In complete darkness, the ideal position was one which elicited a maximum illusion of horizontal rightward displacement of a stationary, centrally presented spot of red light. This location on the neck was marked for later treatment sessions, which were conducted under normal illumination.

\section{Assessment of treatment effects}

Five tests were used to investigate the efficiency of treatment on different aspects of the neglect syndrome.

\section{Subjective visual straight ahead test (SSA)}

The SSA measurement was used to investigate the deviation of the perceived body midline in neglect, and to test for the susceptibility of the patients to neck muscle vibration. Each testing condition started with a spot of light presented pseudorandomly on the horizontal plane at $\pm 10^{\circ}$ in complete darkness on a PC screen. For 10 trials, patients instructed the investigator to move the spot to their estimated subjectively perceived "straight ahead position.." ${ }^{13}$ The mean deviation in degrees of visual angle was measured.

\section{Cancellation test}

Visual exploration was investigated using a cancellation test, a standard clinical method for assessing the ability to scan the entire width of a restricted visual search field. The patient was presented with a high density array of 224 geometric stimuli (144 distractors and 80 targets) on a $29.7 \times 42 \mathrm{~cm}$ sheet of paper. The task was to cancel out the 80 targets with a pencil using the right hand. There were no time restrictions. The number of targets detected was measured.

\section{Tactile search}

This task was performed to investigate a possible crossmodal transfer effect of both treatments on the scanning behaviour of a restricted non-visual search field. The blindfolded subjects had to explore the surface of a horizontal semicircular board (radius $50 \mathrm{~cm}$ ) in peripersonal space. The surface consisted of 72 geometric stimuli (squares, circles, and triangles), 48 with a rough and 24 with a smooth surface, which were equally distributed across four sectors. The exploration started with the index finger positioned at the lower centre of the table and targets, consisting of 24 squares with a rough surface, were identified by using the thumb and index finger of the right hand. The patients had to inform the examiner verbally when they encountered a target. It had been established in a previous training phase that patients were readily able to discriminate the form and the surface of the objects. A session consisted of two search trials. The examiner noted the number of correctly detected targets.

\section{Indented text reading}

To examine therapy transfer to a non-trained visual task and to include an important daily life activity, an indented paragraph reading test was performed. Six parallel versions
(270 to 275 words; arial font, point size 12; double line spacing) of a meaningful text-printed on a $21 \times 29.7 \mathrm{~cm}$ sheet of paper-were used in a random order across the measurements. The 21 to 24 lines were variably indented 0 to 25 spaces from the left and right margin. The patients were instructed to read the text aloud as accurately as possible without using their finger. The number of all correctly read words was recorded.

\section{Visual size discrimination}

To investigate neglect behaviour not associated with visuomotor activity, a horizontal visual size estimation task was performed. ${ }^{39}$ A horizontal bar $(3 \times 1 \mathrm{~cm})$ displayed on a computer monitor was presented on the left side and had to be adjusted to match the horizontal length of a longer bar $(6 \times 1$ $\mathrm{cm})$ presented on the right side. Subjects achieved the match by verbally instructing the experimenter to provide the appropriate adjustments until the lengths appeared equal (20 trials). The mean percentage deviation from the reference rectangle was determined.

\section{Questionnaire for everyday problems of visuospatial deficits}

To obtain supplementary information on potential treatment effects on neglect related daily life problems, a modified and extended German version of the questionnaire of Towle and Lincoln ${ }^{40}$ was administered. The questionnaire consisted of 30 items which were grouped into six subscales attributed to the following problem categories: personal care; reaching and grasping; spatial orientation; time orientation, awareness of the deficit. To prevent any observational bias on the examiner's part, the patient's relatives or the hospital staff (if the patient was a hospital inpatient) recorded how often every problem occurred with respect to each item. The scoring used was as follows: 0 , never; 1 , rarely; 2 , sometimes; 3 , often.

\section{Procedure}

During therapy and neglect assessment the performance was always monitored with the sagittal plane of the head and body of the patient aligned to the objective centre of the given stimulus array. Head position was either fixed with a chin rest or controlled manually. No task was time restricted. All PC based tasks were performed on a 17 inch monitor at a distance of $30 \mathrm{~cm}$.

Every patient received both standard and combined treatments in a crossover design (fig 1). To distinguish therapeutic effects from spontaneous recovery, treatment was preceded by a baseline period of three weeks. After the baseline period, half the patients received 15 sessions of the visual exploration training. In the second phase ( 15 sessions) this treatment was combined with neck muscle vibration (standard-combination; group SC). The other half of the treatment group received the treatments in reverse order (combination-standard; group CS). With the exception of the questionnaire, performance was measured six times: three baseline measurements, two post-treatment measurements, and one follow up measurement after two months. The questionnaire was administered four times during the study: during the first baseline measurement (B1), after each treatment phase $(\mathrm{Tl} / \mathrm{T} 2)$, and after the follow up interval (F).

\section{Statistical analysis}

The changeover time in performance on the different neglect tasks was analysed by a series of four separate repeated measurement analyses of variance (ANOVA), based on the group means at each time of measurement. First, the three baseline measurements between the two therapy groups were compared by means of a two way ANOVA (group $\times$ baseline). Then in a second step we examined the treatment effects by performing an ANOVA with the independent factor treatment 


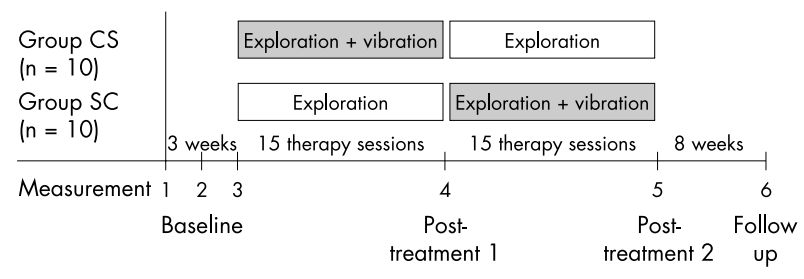

Figure 1 Crossover study design.

sequence (combined treatment first $v$ standard treatment first) and the within-factor time of measurement (mean baseline $v \mathrm{~T} 1 v \mathrm{~T} 2$ ). This ANOVA was redone using the mean baseline values for covariance analysis (ANCOVA).

The follow up was compared with the last post-treatment measurement by means of a two way ANOVA (treatment sequence $\times$ time of measurement). The Greenhouse-Geisser correction to degrees of freedom was applied if appropriate.

For pairwise within-group comparisons, $t$ tests for matched samples were used (two tailed, significance threshold set at $\mathrm{p}<0.01)$. Independent $t$ tests for between-group comparisons were performed to address the treatment effects more directly: pre-post differences were calculated for each task and for each of the two treatment phases. Effects were calculated as differences of post-treatment 1 minus mean baseline, posttreatment 2 minus post-treatment 1 , and follow up minus mean baseline, respectively. These values were used to compare the treatment benefits of the two groups on each period and the therapy gains with respect to the ipsilesional and contralesional sides of the search field. In addition, the relation between the treatment gains of the combination therapy and the follow up difference values were analysed by a series of bivariate correlational analyses (Pearson).

We also re-examined the difference values of both treatments for each task, with respect to the treatment sequences, by means of specific non-parametric rank tests for a two period crossover design (for details, see Brunner and Neumann ${ }^{41}$ ) to compare the treatment effects with respect to carry over and period effects. As the results of this analysis were indistinguishable from the results of our previous statistics, we only report the results of the latter.

For the subscales of the activities of daily living (ADL) questionnaire we obtained the mean scores of each time of measurement. These were used to compute the difference scores following the above procedure. With regard to the ordinal data and the supplementary nature of the questionnaire, we restricted our analysis to pairwise comparisons using nonparametric tests (scalewise). Wilcoxon tests were applied to compare the mean change in performance of each treatment, and the last treatment measurement with the follow up measure. Mann-Whitney U tests were used to examine the differential treatment gains of the two groups on each treatment phase. Because of the more conservative nonparametric methods the significance threshold was set at $\mathrm{p}<0.05$.

\section{RESULTS}

\section{Subjective visual straight ahead test} Pretreatment

In the testing condition searching for the optimal position of the vibrator on the individual's neck, we achieved an illusion of rightward horizontal displacement of the centrally presented fixation point in every subject. Accordingly, the SSA position shifted leftwards during vibration. The displacement (mean) with respect to the zero meridian varied in group CS from $-5.03^{\circ}$ to $-10.11^{\circ}(7.45)$ and in group SC from $-4.31^{\circ}$ to $-9.6^{\circ}(6,43)$. There were no significant group differences $(t(18)=1.14, \mathrm{p}>0.05)$.
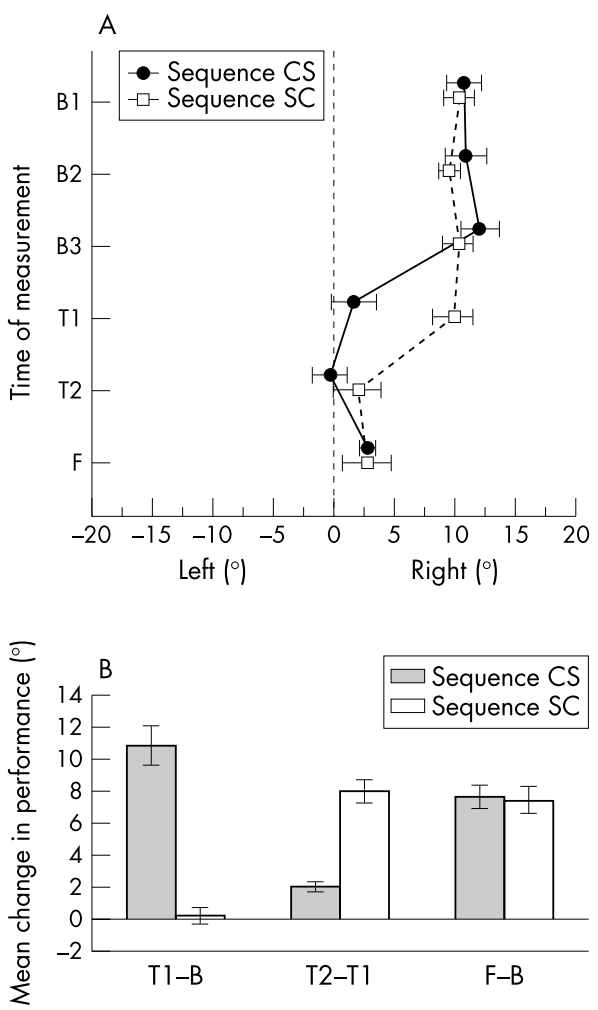

Figure 2 (A) Visual subjective straight ahead judgements. Mean displacements of visual angle from the zero meridian are shown for both treatment groups across baseline (B1-B3), after treatment phases 1 and 2 (T1/T2) and two months after cessation of treatment (F). (B) Mean pre-post differences in treatment phase 1 (T1-mean baseline) and phase 2 (T2-T1), and treatment gains with respect to the mean baseline ( $\mathrm{F}$-mean baseline) according to treatment sequences. Differences are corrected for signs. Error bars = SEM. CS, combination-standard treatment sequence; SC, standard-combination treatment sequence.

\section{Therapy}

During the whole baseline period both groups showed a substantial ipsilesional (rightward) deviation (fig 2A). Comparing the three baseline measurements between the two therapy groups, there were no significant main effects or interactions $(\max \mathrm{F}(2,36)=1.83 ; \mathrm{p}>0.05)$. Hence, for outcome analysis the mean (SEM) of the three baseline measurements in each group was used (group CS, $10.34(1.58)^{\circ}$; group SC, 10.12 $\left.(0.94)^{\circ}\right)$.

The analysis of the change in performance between the mean baseline and the two post-treatment measurements revealed a significant main effect of time of measurement $(\mathrm{F}(2,28)=24.90 ; \mathrm{p}<0.0001)$ and an interaction with treatment sequence $(\mathrm{F}(2,28)=12.10 ; \mathrm{p}<0.0001)$. In contrast, we found no main effect for treatment sequence $(F(1,18)=3.53$; $\mathrm{p}=0.077)$. The differential treatment gains were not influenced by different baseline levels in the two groups $(\mathrm{F}(1,17)=0.01 ; \mathrm{p}=0.915$, ANCOVA $)$.

As illustrated in fig 2A the initial straight ahead deviation was clearly reduced after the combined treatment in both treatment groups. After the first treatment phase (T1), the deviation significantly decreased only in the group receiving the experimental treatment and not in the group receiving standard treatment $(t(9)=3.54, \mathrm{p}=0.006 \vee t(9)=0.21$, $\mathrm{p}=0.836$ ). After the second therapy phase (T2), the group that now received combined treatment (SC) also showed a substantial reduction in the deviation $(t(9)=5.19$; $\mathrm{p}=0.001)$. A further improvement was also observed in the group now receiving standard treatment (CS) which slightly deviated to the left (mean $-0.41 \quad(1.5)^{\circ} ; t(9)=2.70$; 

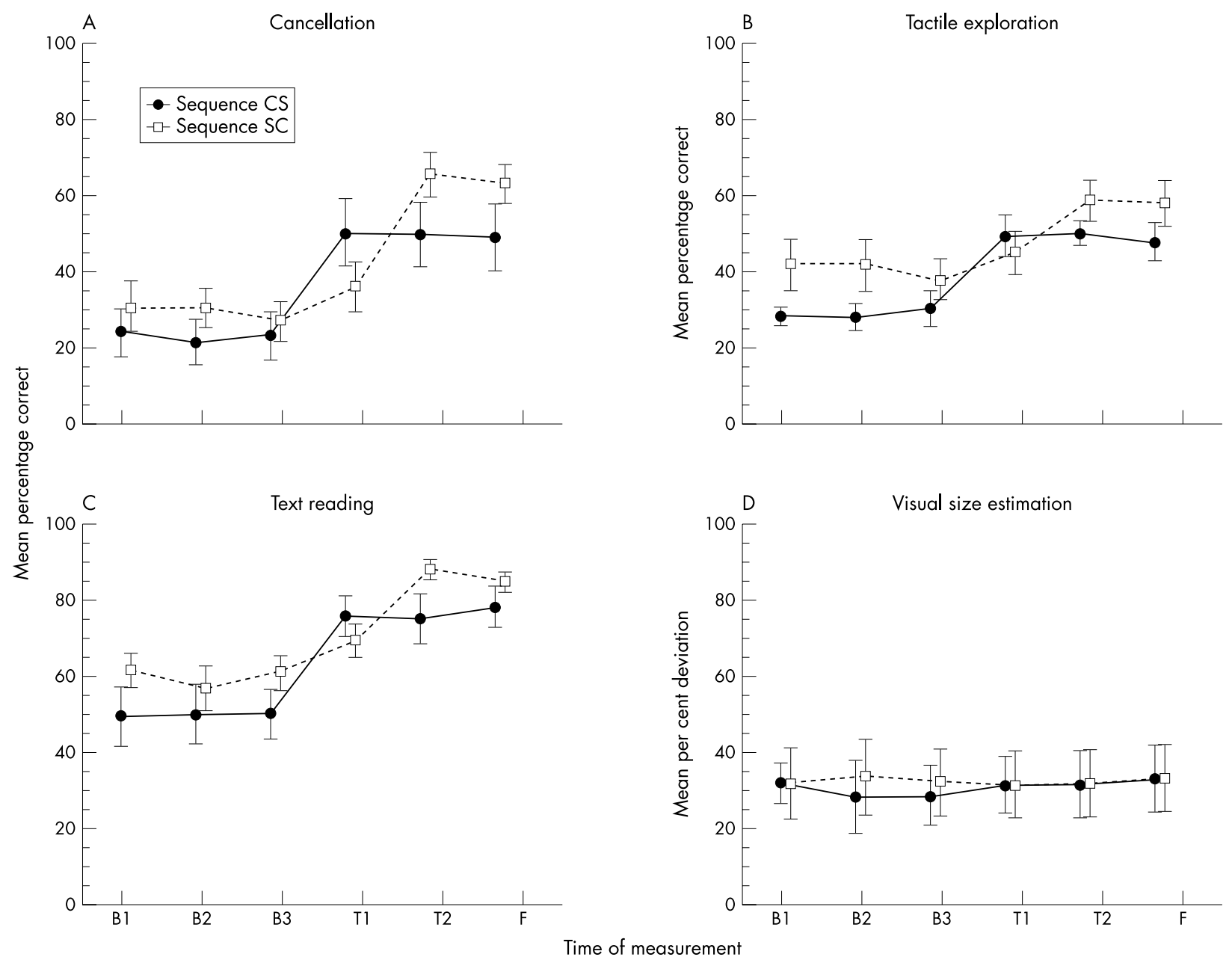

Figure 3 Results of the neglect assessment for the two treatment sequences. The mean percentage of all hits in both groups across baseline $(B 1-B 3)$, treatments $(T 1-T 2)$, and follow up $(F)$ is shown for $(A)$ cancellation, $(B)$ tactile exploration, and $(C)$ text reading. For visual size estimation the mean percentage deviation is shown (D). Error bars = SEM.

$p=0.023$ ), thus indicating an after effect of the previous combined treatment. Between-group comparisons for the two treatment sequences always revealed larger reductions in the SSA deviation in the group that received combined therapy compared with the standard treatment (mean $7.92(1.53)^{\circ}$ to $10.76(2.23)^{\circ} v$ mean $0.22(1.03)^{\circ}$ to $2.11(0.77)^{\circ} ; t(18)=4.23$, $\mathrm{p}<0.0001$; see also fig $2 \mathrm{~B})$.

Two months post-treatment, a moderate increase in the rightward deviation of the group CS could be observed. However, there was no significant main effect or interaction between the last treatment measurement, follow up, and the treatment sequence $(\max F(1,18)=0.43, p>0.50)$. As shown in fig $2 \mathrm{~B}$, the treatment gains remained stable with respect to the mean baseline independently of the treatment sequence (mean $7.39(1.66)^{\circ}$ to $\left.7.60(1.43)^{\circ}\right)$.

\section{Neglect tests}

\section{Baseline}

The graphs in fig 3 suggest better baseline performance for group SC than for group CS for cancellation, tactile exploration, and text reading. Nevertheless, the separate ANOVAs revealed neither significantly different main effects nor interactions $(\max F(1,18)=3.05 ; p>0.05)$. Hence the outcome analysis was also based on the mean of the three baseline measurements in each group for these tasks.

\section{Outcome}

The analyses of the change in performance over time between the mean baseline and the two post-treatment measurements revealed a significant main effect of time of measurement and a significant interaction with treatment sequence, both for cancellation and for tactile exploration and text reading (min $\mathrm{F}(2,36)=6.60 ; \mathrm{p}<0.05)$. The main effect for treatment sequence was not significant $(\max F(2,36)=0.96 ; p<0.05)$. The covariance analyses excluded the possibility that differential treatment gains were related to different baseline levels in the two groups $(\max F(1,17)=0.39 ; \mathrm{p}>0.05)$.

The within-group comparisons for the above mentioned tasks revealed a similar pattern of treatment effects over time (fig 3, panels A to C): for each group, the number of omissions was reduced with respect to the preceding measure only after the combined treatment $(t(9)=-3,19, \mathrm{p}<0.05)$. In contrast, the standard treatment failed to produce any significant effect on either treatment group in the second treatment phase $(t(9)=-2,14, \mathrm{p}>0.05)$. Figure 4 focuses on these results, illustrating the mean pre-post differences of treatments, with SEM, for each task and treatment sequence. For all tasks, between-group comparisons of the two treatment sequences revealed significantly higher therapy gains after combined treatment $(13.5(4.2) \%$ to $29.5(2.16) \%)$ compared with the standard treatment $(0.9(3.6) \%$ to $9.7(4.5) \%)(t(18)$ between -8.09 and 5.86, $\mathrm{p}<0.05)$.

Unlike exploration and reading, the deteriorated size estimation persisted in both patient groups: no treatment method led to a substantial decrease in the mean percentage deviation from the reference rectangle $(\max \mathrm{F}(2,36)=0.53$; $\mathrm{p}>0.05)$. 

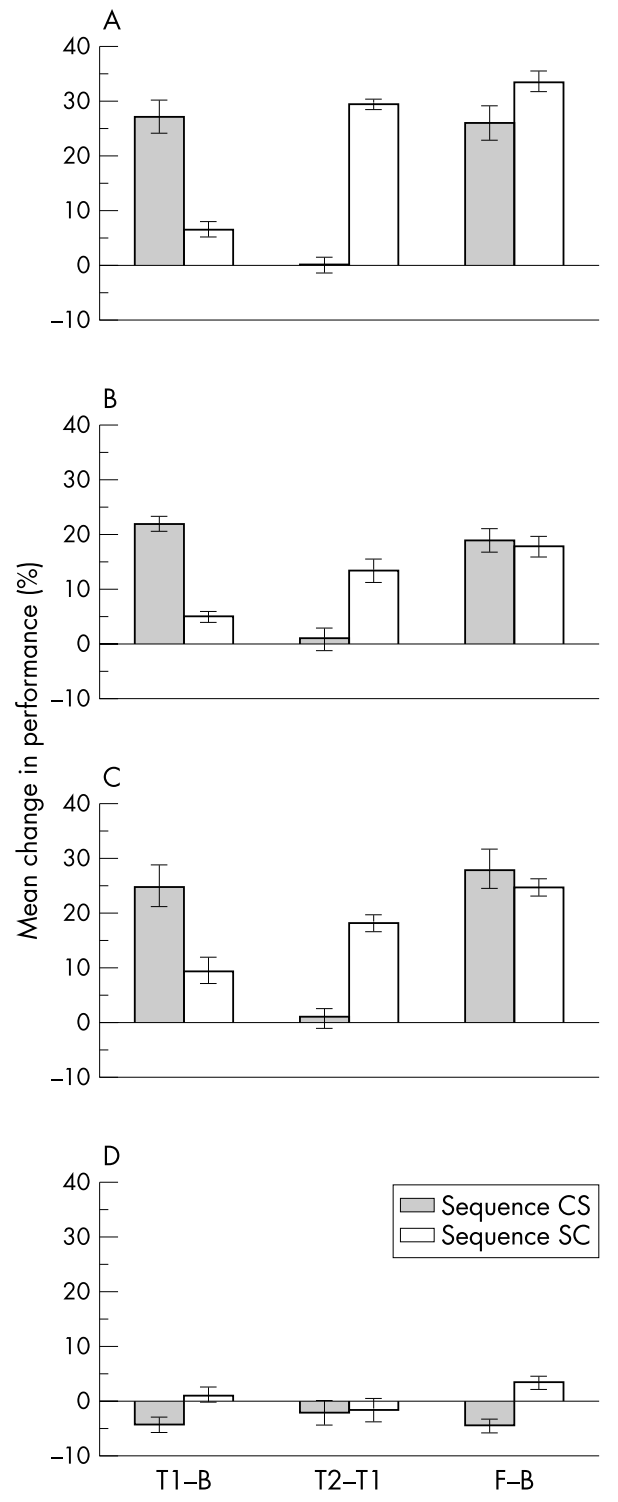

Figure 4 Mean pre-post differences of treatment phase 1 ( T1-mean baseline) and phase 2 (T2-T1), and treatment gains with respect to the mean baseline ( $F$-mean baseline) according to treatment sequences. Error bars $=$ SEM

In sum, these results suggest transfer effects of the combined treatment with respect to non-trained activities of daily living (text reading) and exploration in a non-visual mode (tactile search). In contrast, the effects of the pure exploration training were limited to the visual mode and to the first treatment period.

\section{Follow up}

Comparing the follow up with the last post-treatment measurement, the ANOVAs failed to reveal significant main effects or interactions in any task $(\max F(1,18)=2.57$; $\mathrm{p}>0.05$ ). Thus all treatment effects remained stable after the two months follow up.

\section{Treatment gains within the search fields}

Figure 5 shows the effects of both treatments and their stability in the follow up interval with respect to the ipsilesional and contralesional sides of the search field. The values were based on the same pre-post differences as in the previous analyses but represent the overall mean treatment gain.

In cancellation, tactile exploration, and text reading, the additive effects of vibration were significantly higher than

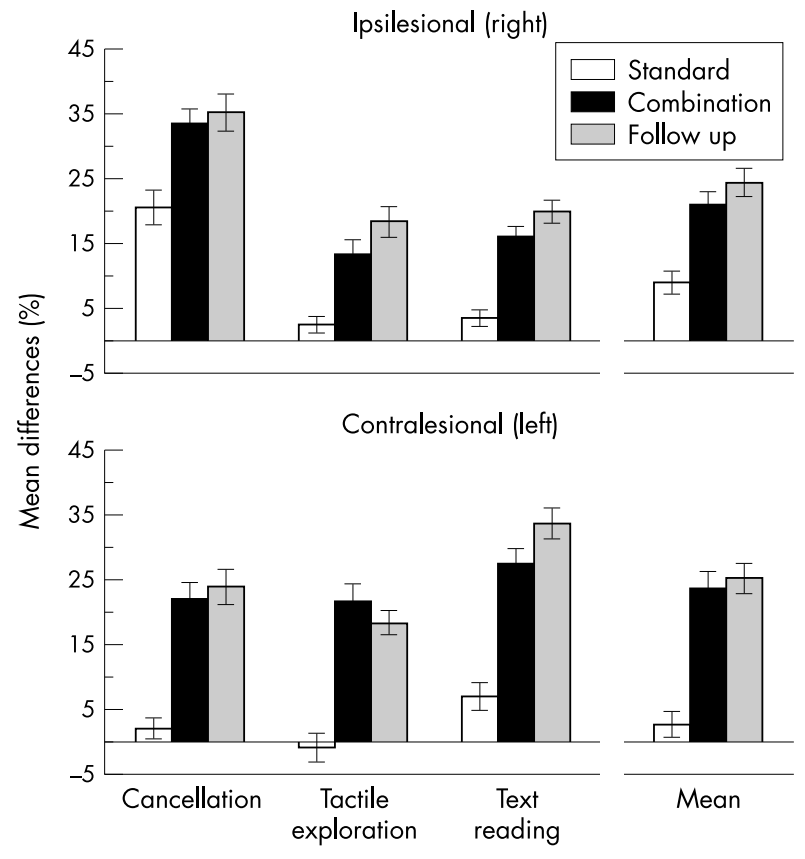

Figure 5 Mean effects for standard treatment and combined treatment across the exploration tasks and text reading. Effects were calculated as differences of T1-mean baseline, T2-T1, and F-mean baseline, respectively. The results are presented with respect to the ipsilesional (right) side or contralesional (left) side of the stimulus array. Error bars $=$ SEM.

with the standard treatment, regardless of the side of the search field $(t(19)=3.72, \mathrm{p}<0.005)$. On the left side, the mean (SEM) percentage of "targets detected" or "words read" changed between $-0.9(4.4) \%$ and $7.0(4.0) \%$ after the standard treatment and between $21.8(5.0) \%$ and $27.2(5.3) \%$ after the combined therapy. On the right side the range was 2.6 $(2.8) \%$ to $21(5.17) \%$ for the standard treatment and 13.4 (4.7)\% to 33.7 (4.2)\% for the combined therapy.

Comparing the treatment gains on the right and left side separately for each therapy method and task, we found only significantly larger effects for text reading on the contralesional side after combined therapy and for cancellation on the ipsilesional side after the standard treatment $(t(19)=-3.65$ and 3.37, $\mathrm{p}<0.005 \vee t(19)=1.47, \mathrm{p}>0.05)$. At follow up, for both search fields only minor changes $(0.26$ $(0.94) \%$ to $2.5(3.62) \%)$ with respect to the last treatment were observed $(t(19)=-1.26 ; \mathrm{p}>0.05)$. Consequently, the treatment gains with respect to the mean baseline (SEM) were between $18.5(4.6) \%$ and $35.0(5.5) \%$ on the ipsilesional (right) side, and between $18.4(3.4) \%$ and $34.0(4.8) \%$ on the contralesional (left) side of the search fields.

\section{ADL questionnaire}

Figure 6 illustrates the mean change of the rating scores with respect to the subscales of the ADL questionnaire. Positive/ negative values indicate a decrease/increase in the frequency of the observed everyday problems of the patients. The separate pairwise comparisons between the pre-post differences of both treatments showed a significant improvement for the subscales "personal care," "reaching and grasping," and "spatial orientation" after the combined treatment compared with the standard treatment $(z \leqslant-2.45, \mathrm{p}<0.05)$. In contrast, no differences in the treatment gains of either therapy method were found for orientation in time $(z=-1.27$, $p>0.05$ ) and only a tendency towards higher treatment gains of the combined treatment for awareness of the deficit was observed $(\mathrm{z}=-1.98, \mathrm{p}=0.05)$.

Comparing the results of the last treatment measure with the follow up measure, no group showed any significant 


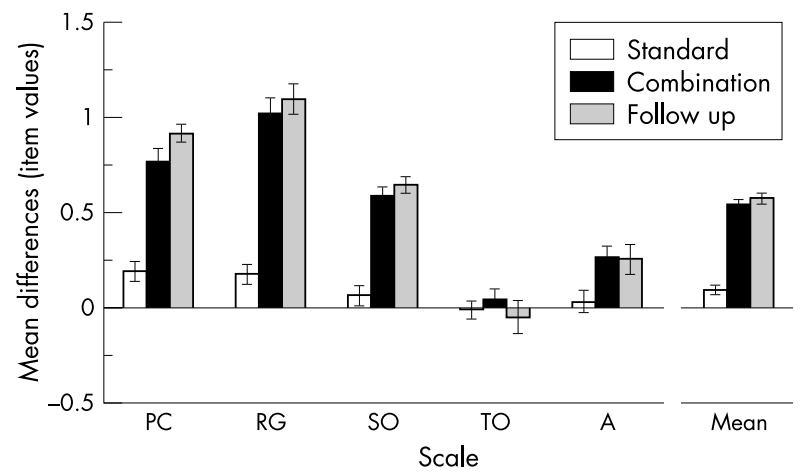

Figure 6 Activities of daily living (ADL) questionnaire. Mean effects for standard treatment and combined treatment across the subscales personal care $(P C)$, reaching and grasping (RG), spacial orientation (SO), orientation in time (TO), and awareness of the deficit (A). Effects were calculated as differences of T1-baseline, T2-T1, and

$\mathrm{F}$-baseline, respectively. Error bars $=$ SEM.

changes in any of the subscales $(z \geqslant-1.55, p>0.05)$. This is reflected in the difference scores between the follow up interval and the mean baseline as shown in fig 6. The separate between group comparisons of the treatment gains confirmed the positive results for the scales "personal care," "reaching and grasping," and "spatial orientation." For these subscales, significant treatment effects were only found for conditions where vibration was added to the exploration training, whereas no significant differences were obtained for the remaining subscales (TO, A) $(\mathrm{U} \leqslant 22.50, \mathrm{p}<0.05 \quad v$ $U \geqslant 26.50, p>0.05$ ).

\section{Vibration effect and treatment gains}

A series of correlational analyses was conducted to investigate the relation between the treatment gains in the neglect tasks and the individual susceptibility to the vibration stimulation. For this purpose we correlated the overall treatment effects of the combination therapy on SSA, cancellation, tactile exploration, and reading with the pretreatment SSA deviation during vibration $\left(S_{S A}\right)$. Horizontal size estimation was excluded from this analysis as no treatment benefits were observed. First, the intercorrelations between the treatment gains on the neglect tasks were examined. No significant correlations were found ( $r=0.39, \mathrm{p}>0.05$ in all cases), suggesting that these tasks were measuring different aspects of neglect. Nonetheless, for all tasks a significant correlation between $\mathrm{SSA}_{\mathrm{V}}$ and the treatment gains of the combination treatment was found (SSA: $r=0.86$; cancellation: $r=0.57$; tactile exploration: $r=0.55$; reading: $r=0.52 ; \mathrm{p}<0.05$ in all cases $)$.

Interestingly the difference values between the final treatment measure and the follow up assessment did not correlate significantly with $\mathrm{SSA}_{\mathrm{v}}(r=0.058, \mathrm{p}>0.5$ in all cases $)$, indicating that the long term stability of the treatment effect could not be predicted from the initial susceptibility to vibration. However, although the majority of the intercorrelations between the neglect tasks remained non-significant, weak but significant correlations were found between the SSA and the neglect tasks (cancellation: $r=0.49$; tactile exploration: $r=0.5 \mathrm{l}$; reading: $r=0.47$; all $\mathrm{p}<0.05$ ). This pattern of outcomes may suggest that the recalibration of the body sagittal plane does, to some extent, determine the long term stability of the treatment gains across a wider range of clinical tasks.

\section{DISCUSSION}

In this crossover study we investigated the differential treatment effects of visual exploration training alone and visual exploration training in combination with neck muscle vibration. The results provide clear evidence that the addition of contralesional neck vibration to a standard method of treating neglect leads to additive and lasting treatment effects. Further, we showed that the additive effects of vibration transferred from trained tasks (visual exploration) to untrained tasks (text reading), and from the visual to the tactile mode. Moreover, the combined treatment led to a reduction in daily life problems concerning self care, reaching and grasping, and spatial orientation in comparison with the effects achieved by exploration training alone.

These improvements are not the result of therapeutic "cost effects" because in the exploration tasks as well as in text reading the amelioration of exploration behaviour on the contralesional left side was not accompanied by an increase of omissions on the ipsilesional right side. The observation of increased improvement on the right side of the search field can be explained by the degree of severity of neglect in our patient sample. Although the exact nature of the distribution of the orientational bias in neglect remains a matter of debate, it is well established that marked neglect can affect the ipsilesional as well as the contralesional half space. This was reflected by the observation that before treatment onset a majority of our severe neglect patients showed a strong tendency to explore only the extreme right side of the stimulus display, regardless of the mode in which the stimuli were presented.

The observed improvements also cannot be attributed to practice effects or to non-specific cueing and arousal by vibrotactile stimulation or the noise elicited by the vibrator. First, we used different tasks for treatment and assessment. Second, the specificity of neck vibration has been shown in former studies, which found no beneficial effects of contralesional (left) hand vibration or the noise of the vibrator on neglect symptoms. ${ }^{17}{ }^{42}$ Third, our own results showed differential effects with respect to different treatment periods and neglect tasks. In particular, the perceptual size distortion, which is often associated with neglect, was not affected by vibration. One possible explanation is that the nature of this task demands an allocentric reference system, which is dissociable from egocentric representations. ${ }^{43}$

The observed effects of neck muscle vibration on visual and tactile exploration in neglect fit well with the idea that vibration manipulates an egocentric reference system for spatiomotor transformation by generating a corrective head-ontrunk signal. ${ }^{44}$ In a series of experiments on normal subjects

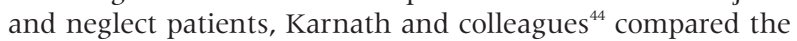
effects of caloric stimulation with contralesional neck muscle vibration. They demonstrated that both methods led to a similar shift in the distribution of exploratory eye movements in the direction of the side of stimulation. From this it was concluded that both stimulation methods influence cortical structures in which vestibular and proprioceptive signals converge with afferent inputs from other modalities to build egocentric representations of outer space.

The specificity of the vibration effect might also explain why our neglect patients still showed moderate neglect phenomena after combined training in the search tasks and in text reading. Even if more extended treatment sessions with vibration were to yield larger effects, other factors are very likely to contribute to neglect. With respect to the extended lesions of most of our patients we cannot exclude the possibility that intentional impairments, ${ }^{45}$ generalised hypoarousal, ${ }^{11}$ imbalanced interactions between global and local attentional processes, ${ }^{46}$ or even working memory deficits $^{47}$ contributed to the residual neglect phenomena and possibly require additional specific treatment. ${ }^{48} 49$

The relatively small benefits of the neglect patients following the isolated exploration training might result from the fact that one essential aspect of this treatment is to learn compensatory strategies. In cases of severe neglect, the ability to acquire such strategies is seriously limited, requiring between 20 and 40 training sessions to obtain measurable effects. ${ }^{56}$ 
Consequently, the emergence of beneficial effects requires longer periods of time than were allowed for in the present study. In this respect the criticism might be raised that our design lacked fairness in that only 15 sessions were assigned to each treatment. However, the therapeutic effects of exploration training were mainly observed in the first treatment period and not in the second. Further, as exploration therapy focuses on improving the symptom of disturbed visual scanning, it probably fails to ameliorate the crucial underlying problem of distorted spatial representation or attentional deficits.

\section{Conclusions}

Vibration of the contralesional neck muscles offers considerable potential for specific neglect rehabilitation, leading to a lasting and more efficient reduction of neglect symptoms than is obtained by standard treatments. Neck muscle vibration can be carried out in parallel with other treatments and requires low cost vibration technology, such as that used in physiotherapy. It is well suited for clinical use as an ideal add-on technique in neglect rehabilitation.

\section{ACKNOWLEDGEMENTS}

We wish to thank two anonymous referees for their comments on an earlier version of this manuscript.

\section{Authors' affiliations}

I Schindler, G Kerkhoff, G Goldenberg, EKN-Clinical

Neuropsychology Research Group, Department of Neuropsychology, City Hospital Bogenhausen, Dachauer Str, Munich, Germany H-O Karnath, Department of Cognitive Neurology, University of Tübingen, Hoppe-Seyler-Str, Tübingen, Germany

I Keller, Neurological Clinic Bad Aibling, Kolbermoorerstr 72, Bad Aibling, Germany

Competing interests: none declared.

\section{REFERENCES}

1 Driver J, Mattingley JB. Parietal neglect and visual awareness. Nat Neurosci 1998;1:17-22.

2 Robertson IH, Eglin M. Attentional search in unilateral visual neglect. In: Robertson IH, Marshall JC, eds. Unilateral neglect: clinical and experimental studies. Hove: Lawrence Erlbaum Associates Publishers, 1993: 169-91.

3 Stone SP, Patel P, Greenwood RJ, et al. Measuring visual neglect in acute stroke and predicting its recovery: the visual neglect recovery index. J Neurol Neurosurg Psychiatry 1992;55:431-6.

4 Antonucci G, Guariglia C, Judica A, et al. Effectiveness of neglect rehabilitation in a randomized group study. J Clin Exp Neuropsychol 1995; 17:383-9.

5 Kerkhoff G. Rehabilitation of visuospatial cognition and visual exploration in neglect: a cross-over study. Restor Neurol Neurosci $1998 ; 12: 27-40$.

6 Pizzamiglio L, Antonucci G, Judica A, et al. Cognitive rehabilitation of the hemineglect disorder in chronic patients with unilateral right brain damage. J Clin Exp Neuropsychol 1992;14:901-23.

7 Kerkhoff G. Multiple perceptual distortions and their modulations in leftsided neglect. Neuropsychologia 2000;38: 1073-86.

8 Paolucci S, Antonucci G, Guariglia C, et al. Facilitatory effect of neglect rehabilitation on the recovery of left hemiplegic stroke patients: a cross-over study. J Neurol 1996;243:308-14.

9 Weinberg J, Diller L, Gordon W, et al. Visual scanning training effect on reading-related tasks in acquired right brain damage. Arch Phys Med Rehab 1977;58:479-86.

10 Wagenaar RC, Van Wieringen PCW, Netelenbos JB, et al. The transfer of scanning training effects in visual attention after stroke: five single case studies. Disabil Rehabil 1992;14:51-60.

11 Robertson IH, Tegnér R, Tham K, et al. Sustained attention training for unilateral neglect: theoretical and rehabilitation implications. J Clin Exp Neuropsychol 1995;17:416-30.

12 Wilson FC, Manly T, Coyle D, et al. The effect of contralesional limb activation training and sustained attention training for self-care programmes in unilateral spatial neglect. Restor Neurol Neurosci 2000; 16:1-4

13 Rode G, Perenin MT. Temporary remission of representational hemineglect through vestibular stimulation. Neuroreport 1994:5:869-72.

14 Rubens AB. Caloric stimulation and unilateral visual neglect. Neurology 1985;35:1019-24

15 Pizzamiglio L, Frasca R, Guariglia C, et al. Effect of optokinetic stimulation in patients with visual neglect. Cortex 1990;26:541-54.
16 Vallar G, Guariglia C, Magnotti L, et al. Optokinetic stimulation affects both vertical and horizontal deficits of position sense in unilateral neglect. Cortex 1995;31:669-83.

17 Karnath HO, Christ K, Hartje W. Decrease of contralateral neglect by neck muscle vibration and spatial orientation of trunk midline. Brain 1993; 1 16:383-96.

18 Karnath HO, Fetter M, Dichgans J. Ocular exploration of space as a function of neck proprioceptive and vestibular input: observations in normal subjects and patients with spatial neglect after parietal lesions. Exp Brain Res 1996;109:333-42.

19 Frassinetti F, Angeli V, Meneghello F, et al. Long-lasting amelioration of visuospatial neglect by prism adaptation. Brain 2002;125:608-23.

20 Rossetti Y, Rode G, Pisella L, et al. Prism adaption to a rightward optical deviation rehabilitates left hemispatial neglect. Nature 1998;395: 166-9.

21 Kalra L, Perez I, Gupta S, et al. The influence of visual neglect on stroke rehabilitation. Stroke 1997:28:1386-91.

22 Robertson IH, North N, Geggie C. Spatiomotor cuing in unilateral left neglect: three case study of its therapeutic effects. J Neurol Neurosurg Psychiatry 1992;55:799-805.

23 Samuel C, Louis-Dreyfus A, Kaschel R, et al. Rehabilitation of very severe unilateral neglect by visuo-space cueing: two single case studies. Neuropsychol Rehabil 2000;10:385-99.

24 Robertson IH, Hogg K, McMillan TM. Rehabilitation of unilateral neglect: improving function by contralesional limb activation. Neuropsychol Rehabil 1998;8:19-29.

25 Wiart L, Bon-Saint-Côme A, Debelleix X, et al. Unilateral neglect syndrome rehabilitation by trunk rotation and scanning training. Arch Phys Med Rehabil 1997;78:1-6.

26 Karnath HO, Reich E, Rorden C, et al. The perception of body orientation after neck-proprioceptive stimulation: effects of time and of visual cueing. Exp Brain Res 2002;143:350-8.

27 Ferber S, Bahlo S, Ackermann H, et al. Vibration der Nackenmuskulatur als Therapie bei Neglectsymptomatik? - Eine Fallstudie. Neurol Rehabil 1998:4:21-4.

28 Vallar G, Perani D. The anatomy of unilateral neglect after right-hemisphere stroke lesions. A clinical/CT-scan correlation study in man. Neuropsychologia 1986;24:609-22.

29 Andersen RA, Lawrence $\mathrm{H}$, Snyder $\mathrm{LH}$, et al. Multimodal representation of space in the posterior parietal cortex and its use in planning movements. Annu Rev Neurosci 1997;20:303-30

30 Colby CL. Action-oriented spatial reference frames in Cortex. Neuron 1998;20:15-24.

31 Pouget A, Driver J. Relating unilateral neglect to the neural coding of space. Curr Opin Neurobiol 2000;10:242-9.

32 Andersen RA. Coordinate transformations and motor planning in posterior parietal cortex. In: Gazzaniga MS, ed. The cognitive neurosciences. Cambridge, London: The MIT Press 1995:519-32.

33 Karnath HO, Dick H, Konczak J. Kinematics of goal-directed arm movements in neglect: control of hand in space. Neuropsychologia 1997;35:435-44

34 Oldfield RC. The assessment and analysis of handedness: The Edinburgh Inventory. Neuropsychologia 1971;9:97-113.

35 Schenkenberg T, Bradford DC, Ajax ET. Line bisection and unilateral visual neglect in patients with neurologic impairment. Neurology 1980;30:509-17

36 Rapport LJ, Farchione TJ, Dutra RL, et al. Measures of hemi inattention on the Rey Figure copy for the Lezak Osterrieth scoring method. Clin Neuropsychol 1996:10:450-4.

37 Damasio H, Damasio, AR. Lesion analysis in neuropsychology. New York: Oxford University Press, 1989.

38 Biguer B, Donaldson ML, Hein A, et al. Neck muscle vibration modifies the representation of visual motion and direction in man. Brain 1988:111:1405-24.

39 Kerkhoff G, Schindler I, Keller I, et al. Visual background motion reduces size distortion in spatial neglect. Neuroreport 1999;10:319-23.

40 Towle D, Lincoln NB. Development of a questionnaire for detecting everyday problems in stroke patients with unilateral visual neglect. Clin Rehabil 1996:5:135-40.

41 Brunner E, Neumann N. Non-parametric methods for the 2-period-cross-over design under weak model assumptions. Biomed 1987;29:907-20.

42 Karnath HO. Transcutaneous electrical stimulation and vibration of neck muscles in neglect. Exp Brain Res 1995;105:321-4.

43 Milner AD, Harvey M. Distortion of size perception in visuospatial neglect. Curr Biol 1995;5:85-9.

44 Karnath HO. Subjective body orientation in neglect and the interactive contribution of neck muscle proprioreception and vestibular stimulation. Brain 1994:114:1001-12.

45 Mesulam MM. Spatial attention and neglect: parietal, frontal and cingulate contributions to the mental representation and attentional targeting of salient extrapersonal events. Phil Trans $R$ Soc Lond 2002;354:1325-46.

46 Marshall JC, Halligan PW. Seeing the forest but only half the trees? Nature 1995;373:521-3

47 Woiciulik E, Husain M, Clarke K, et al. Spatial working memory deficit in unilateral neglect. Neuropsychologia 2001;39:390-6.

48 Robertson IH, Mattingley JB, Rorden C, et al. Phasic alerting of neglect patients overcomes their spatial deficit in visual awareness. Nature 1998;395: 169-72

49 Robertson IH. Setting goals for cognitive rehabilitation. Curr Opin Neurol 1999:12:703-8. 